\title{
Secondary Fermented Extract of Chaga-Cheonggukjang Attenuates the Effects of Obesity and Suppresses Inflammatory Response in the Liver and Spleen of High-Fat Diet-Induced Obese Mices
}

\author{
Ha Gyoon $\mathrm{Na}^{1 \dagger}$, Yuna Park ${ }^{2 \dagger}$, Min-Ah Kim ${ }^{1 \dagger}$, Jin Woo Lee ${ }^{2}$, Gyeongseop So ${ }^{2}$, Sung Hyeok Kim ${ }^{2}$, Ki-Hyo Jang ${ }^{1}$, \\ Mi-Ja Kim ${ }^{1}$, Seung Namkoong ${ }^{3}$, Hyun Jung Koo ${ }^{4}$, Sung Ryul Lee ${ }^{5 *}$, and Eun-Hwa Sohn ${ }^{2 *}$ \\ ${ }^{1}$ Department of Food and Nutrition, Kangwon National University, Samcheok 25949, Republic of Korea \\ ${ }^{2}$ Department of Herbal Medicine Resource, Kangwon National University, Samcheok 25949, Republic of Korea \\ ${ }^{3}$ Department of Physical Therapy, Kangwon National University, Samcheok 25949, Republic of Korea \\ ${ }^{4}$ Department of Medicinal and Industrial Crops, Korea National College of Agriculture and Fisheries, Jeonju 54874, Republic of Korea \\ ${ }^{5}$ Department of Convergence Biomedical Science, Cardiovascular and Metabolic Disease Center, College of Medicine, Inje University, Busan \\ 47392, Republic of Korea
}

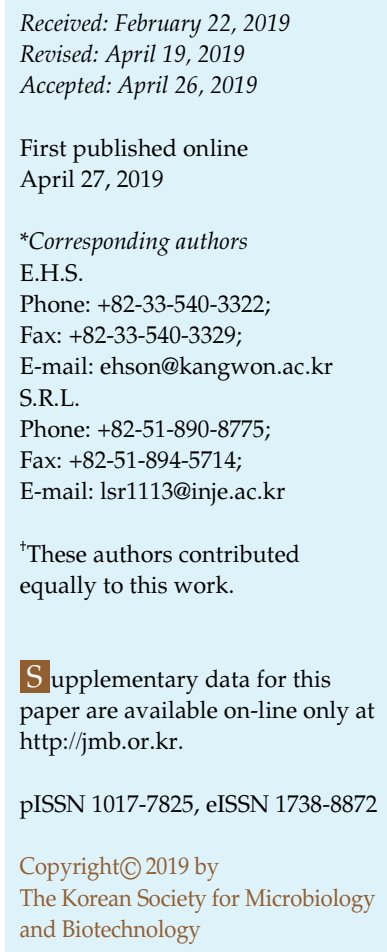

\begin{abstract}
Cheonggukjang and chaga mushrooms have numerous health benefits, and have been used in alternative medicine. Therefore, a powder mixture of 98: Cheonggukjang and 2: Chaga extracts was fermented with Lactobacillus acidophilus KCTC3925 (FCC) and its anti-obesity effects in high-fat diet (HFD)-induced obese mice were determined. Five-week-old male ICR mice were fed a normal diet or HFD in the presence or absence of $3 \%$ and $5 \%$ FCC by weight $(n=10$ per group). After 12 weeks, the mice were sacrificed, and the serum and tissue samples were collected for analysis. Body weight and epididymal fat pad weight were significantly lowered in the $3 \%$ and $5 \%$ FCC groups compared with those in the HFD control group $(p<0.01)$. FCC supplementation suppressed serum triglyceride and increased serum HDL-C levels $(p<0.01)$. Serum GOT, GPT, and leptin levels, hepatic COX-2 mRNA expression, and splenic COX-2 and IL-4 mRNA expression were significantly higher in the HFD groups than in the control group $(p>0.05)$; however, except for splenic IL-4 levels, the increases were significantly attenuated by FCC supplementation. Expression of ICAM-1, an aortic inflammatory marker, was significantly increased in the HFD group; this effect was suppressed in the 3\% FCC group ( $p<$ 0.01 ) but not in the 5\% FCC group. FCC suppressed the body weight and epididymal fat pad weight gain, as well as inflammatory responses in the liver and spleen of HFD-fed mice. Thus, FCC supplementation will be beneficial for the treatment of obesity-related effects.
\end{abstract}

Keywords: Cheonggukjang, chaga mushroom, Lactobacillus acidophilus KCTC3925, high-fat diet, anti-obesity, inflammatory molecules

\section{Introduction}

Overweight and obesity are characterized by a proinflammatory state associated with excessive fat mass and elevated blood lipid profiles [1]. Obesity plays a role in the increased risk of insulin resistance, type 2 diabetes, fatty liver disease, atherosclerosis, degenerative disorders (including dementia), airway disease, and some cancers
[2-4]. In addition to body weight gain due to energy imbalance, the upregulation of various proinflammatory molecules including cyclooxygenase-2 (COX-2), interleukin4 (IL-4), and intercellular adhesion molecule-1 (ICAM-1) is associated with chronic inflammation in obesity $[3,5]$. Despite the immense public health implications of obesity, there has been a marked increase in obesity even among children worldwide [6]. Obesity is typically treated through 
lifestyle modifications such as dietary changes, exercise, and smoking cessation, as well as through pharmacologic intervention with statin therapy, supported by the use of niacin, bile acid sequestrants or cholesterol absorption inhibitors. Though drug therapy can be used for weight loss, no such therapy cures obesity, and weight typically increases shortly after the cessation of drug therapy [7]. Moreover, the use of existing anti-hyperlipidemic drugs is limited in normolipidemic, normoglycemic or normotensive people who are approaching the risk levels. Statins also have several limitations including treatment resistance, intolerance due to adverse events, and a lack of adherence, all of which contribute to poor outcomes [8]. It should be noted that obesity-associated complications are not mediated by a single cause or signaling pathway but by multiple routes [7]. Thus, there is an imperative need for preventative and therapeutic strategies [7].

Soybeans are rich in proteins and contain functional components such as isoflavones, phytic acid, trypsin inhibitor, saponins, phytosterol and phenolic compounds, dietary fiber, and oligosaccharides [9-12]. Cheonggukjang (also called natto, tempeh, and douchi in other Asian regions) is a century-old Korean soybean-based fermented food, that only takes 1-3 days for optimal fermentation, unlike other soybean pastes/soy sources that require a few months or years [13]. Cheonggukjang intake can improve plasma lipid profiles and fasting blood glucose levels in subjects with impaired fasting glucose control [14]. In addition, cheonggukjang intake produces an inhibitory effect on allergic asthma that is exacerbated by histamine release from mast cells, as well as other allergic responses $[15,16]$.

Like soybeans, mushrooms contain enormous amounts of bioactive compounds, including polysaccharides, fibers, terpenes, polyphenols, sterols, flavonoids, and alkaloids [4]. Amongst them, the chaga mushroom (Inonotus obliquus) has been used as a folk medicine in East Asian countries and Russia for centuries [17]. Chaga can be used in combination with other herbs to alleviate gastric and duodenal ulcers, and gastritis [18].

Among the various technological options for food processing, the fermentation of natural plants or their products is an excellent choice for reducing the levels of allergy-causing materials and enriching the bioavailability of beneficial compounds [19-21]. To determine whether the combination of chaga and cheonggukjang would cause weight loss in obese mice, we prepared a secondary fermented extract of cheonggukjang mixed with chaga mushroom extract (Chaga-Cheonggukjang) through fermen- tation with Lactobacillus acidophilus KCTC 3925, referred to herein as FCC [22]. The consumption of a high-fat diet (HFD) causes a positive energy balance in the body and leads to an increase in visceral fat deposition [23]. After mixing commercial HFD chow with 3\% (FCC3) and 5\% FCC (FCC5) by weight, FCC3 and FCC5 were prepared as pellets, and were made freely accessible to mice in meals to minimize interventional stress. Then, we evaluated the effects of 12-week FCC3 and FCC5 intake on HFD-induced obesity parameters (body weight, epididymal fat pad weight, and lipid profiles) and inflammatory responses in the liver (COX-2 mRNA levels), spleen (IL-4 and COX-2 mRNA levels), and aorta (protein level of ICAM-1).

\section{Materials and Methods}

\section{Chemicals and Reagents}

Normal chow diet (D12450B; 10\% kcal\% fat) and high-fat diet (D12492; 60\% kcal\% fat) were obtained from Research Diet, Inc. (USA). Primary antibodies against intercellular adhesion molecule-1 (ICAM-1) and $\beta$-actin were purchased from Cell Signaling Technology (USA). Triglycerides (TG) and total cholesterol (TChol), high-density lipoprotein-cholesterol (HDL-C), low-density lipoprotein-cholesterol (LDL-C) assay kits were obtained from Biovision (USA). Aspartate aminotransferase (GOT) and alanine aminotransferase (GPT) activity assay kits and simvastatin (SV) were purchased from Sigma-Aldrich (USA). Chaga-Cheonggukjang is a cheonggukjang containing $2 \%$ (weight/weight) chaga water extract (Primorsky Territory, Russia) and was obtained from the BARI Co., Ltd. (Korea).

\section{Fermentation of Chaga-Cheonggukjang}

Lactobacillus acidophilus KCTC 3925 has shown a strong capacity for converting glycoside to aglycones, such as genistein, daidzein, and glycitein, in fermented soybean paste [22]. L. acidophilus KCTC 3925 was cultured in de Man, Rogosa and Sharpe (MRS) medium (Difco, USA) for $2-3$ days at $37^{\circ} \mathrm{C}$ without agitation. FCC was obtained after secondary fermentation of Chaga-Cheonggukjang at $40^{\circ} \mathrm{C}$ for $48 \mathrm{~h}$ with L. acidophilus KCTC 3925. As previously reported [24], FCC contains $28 \%$ more total isoflavone aglycones than the original Chaga-Cheonggukjang. FCC3 and FCC5, containing $3 \%$ and $5 \%$ (weight/weight) respectively, of FCC in HFD, were prepared as pellets similar to normal chow diet by Raon Bio Co. (Korea). The composition of the experimental diets and their calories per gram are depicted in Table 1. To avoid auto-oxidation of the fat components, experimental foods were stored at approximately $4^{\circ} \mathrm{C}$.

\section{Experimental Animals and Diets}

All animals received humane care. The experimental animal facility and study protocols were approved by the Animal Care and Use Committee of Kangwon National University (KW- 
Table 1. Composition of experimental diets.

\begin{tabular}{|c|c|c|c|c|}
\hline \multirow{2}{*}{ Ingredients $(\mathrm{g} / \mathrm{kg})$} & \multicolumn{4}{|c|}{ Experimental diets } \\
\hline & ND & HFD & FCC3 & FCC5 \\
\hline Casein & 200 & 200 & 200 & 200 \\
\hline L-Cystine & 3 & 3 & 3 & 3 \\
\hline Corn starch & 315 & 0 & 0 & 0 \\
\hline Maltodextrin 10 & 35 & 125 & 125 & 125 \\
\hline Sucrose & 350 & 68.8 & 68.8 & 68.8 \\
\hline Cellulose, BW 200 & 50 & 50 & 50 & 50 \\
\hline Soybean oil & 25 & 25 & 25 & 25 \\
\hline Lard & 20 & 245 & 245 & 245 \\
\hline Mineral mix S10026 & 10 & 10 & 10 & 10 \\
\hline D-calcium phosphate & 13 & 13 & 13 & 13 \\
\hline Calcium carbonate & 5.5 & 5.5 & 5.5 & 5.5 \\
\hline Potassium citrate & 16.5 & 16.5 & 16.5 & 16.5 \\
\hline Vitamin mix V10001 & 10 & 10 & 10 & 10 \\
\hline Choline bitartrate & 2 & 2 & 2 & 2 \\
\hline FD\&C yellow dye \#5 & 0.05 & - & - & - \\
\hline FD\&C blue dye \#1 & - & 0.05 & 0.05 & 0.05 \\
\hline FCC & & & 30 & 50 \\
\hline Total (g) & 1055.05 & 773.85 & 803.85 & 823.85 \\
\hline Calories per gram & 4.057 & 5.243 & 5.220 & 5.203 \\
\hline
\end{tabular}

ND: normal diet, HFD: high-fat diet, FCC; secondary fermented extract of Chaga-cheonggukjang. FCC3; HFD containing 3\% FCC (weight/weight); FCC5; HFD containing 5\% FCC (weight/weight).

161129-1). All experimental procedures were undertaken in compliance with the Guide for the Care and Use of Laboratory Animals (National Institutes of Health, USA) and the National Animal Welfare Law of the Republic of Korea. Five-week-old ICR male mice were obtained from Orient Bio Inc. (Korea). The mice were maintained in a controlled environment of $22 \pm 1^{\circ} \mathrm{C}$ and humidity of $50 \pm 10 \%$ with a $12 \mathrm{~h}$ light-dark cycle. The mice were provided with sterile standard mouse chow and water ad libitum during the acclimation and experimental periods. After acclimation, mice were randomly divided into five groups $(n=10$ per group) and were fed with normal chow diet (ND), HFD, FCC3, or FCC5 for 12 weeks. Simvastain (SV), a lipid-lowering statin drug that is derived synthetically from the fermentation of Aspergillus terreus, was delivered via oral gavage at a dose of $10 \mathrm{mg} / \mathrm{kg}$ body weight for 6 days per week. During the experiment, clinical signs and general appearance were observed twice per week. Weight gain and food intake, as calculated by the collection and weighing of uneaten food, were recorded twice per week.

\section{Biochemical Analysis and Determination of Tissue Weight}

At the end of the experiment, all animals were fasted for $12 \mathrm{~h}$, and blood was collected from the abdominal vena cava under anesthesia. Blood was centrifuged at 3,000 $\times g$ for $10 \mathrm{~min}$ at $4^{\circ} \mathrm{C}$ to obtain serum. The following parameters were analyzed using a Hemavet 950 Analyzer (Drew, USA): serum TG, T-Chol, HDL-C, LDL-C, GOT, and GPT. Serum leptin levels were measured with a leptin assay kit (Sigma, USA) according to the manufacturer's protocol. The liver, spleen, and epididymal fat were carefully excised, cleaned with sterile $0.9 \% \mathrm{NaCl}$ solution, blotted dry with filter paper, and then weighed. The weight of each tissue was expressed as a relative value to the body weight $(\mathrm{kg})$.

\section{Histological Examination}

The liver and epididymal fat tissue were removed. The liver was perfused with $0.9 \%$ normal saline to remove blood, the largest lobule was sectioned and fixed in 10\% formaldehyde. Paraffin-embedded liver and epididymal fat tissue sections $(3 \mu \mathrm{m})$ were stained with hematoxylin and eosin stain (H\&E). Samples were observed and photographed at 200× magnification. Blinded histological analysis was performed by a trained histologist.

\section{Quantitative RT-PCR (qRT-PCR) Analysis of COX-2 and IL-4}

The mRNA expression levels of cyclooxygenase-2 (COX-2) and interleukin-4 (IL-4) were determined using reverse transcription polymerase chain reaction (RT-PCR). Total RNA was extracted from the liver and spleen using the TRIzol reagent (Invitrogen, USA) and processed using a cDNA Synthesis Kit (TAKARA, Japan). A SYBR Master Mix Kit (TAKARA) was used for RT-PCR, and CDNA was amplified using specific COX-2 (forward, 5'CGTAGCAGATGACTGCCCAA-3'; reverse, 5'-CTTGGGGGTCAG GGATGAAC-3'), IL-4 (forward, 5'-ACAGGAGAAGGGACGCCA T-3'; reverse, 5'- GAAGCCCTACAGACGAGCTCA- $\left.3^{\prime}\right)$, and $\beta$ actin (forward, 5'-TGTCCACCTTCCAGCAGATGT-3'; reverse, 5' AGCTCAGTAACAGTCCGCCTAGA-3') primers. Quantitative real-time RT-PCR reactions were performed on a Light Cycler 96 Instrument (Roche, Switzerland). Relative quantitative evaluation of each gene was performed by the comparative cycle threshold method [25].

\section{Immunoblot Analysis}

The aorta of the experimental animals were harvested and washed with PBS, and the red blood cells were removed with Red Blood Cell Lysis Buffer (Sigma-Aldrich, USA). The vascular tissues were lysed with a homogenizer while immersed in PROPREP Protein Extraction Buffer (Intron Biotechnology, Korea) for $1 \mathrm{~h}$ on ice. After centrifugation at $15,000 \times g$ for $30 \mathrm{~min}$ at $4^{\circ} \mathrm{C}$, the lysates were collected and the protein concentration was determined using a protein assay kit (Bio-Rad Laboratories, USA) with bovine serum albumin (BSA) as the standard. Equal amounts of protein were boiled for $5 \mathrm{~min}$, fractionated by $10 \%$ SDSpolyacrylamide gel electrophoresis (PAGE) (Bio-Rad Laboratories), and electrophoretically transferred to a nitrocellulose membrane. The membrane was blocked with 5\% skim milk and incubated with primary antibodies against ICAM-1. The blots were 
Table 2. Changes in body weight and tissue weight of the spleen, liver, and epididymal fat.

\begin{tabular}{|c|c|c|c|c|c|}
\hline \multirow{2}{*}{$\begin{array}{c}\text { Parameters } \\
(n=10 \text { per group })\end{array}$} & \multicolumn{5}{|c|}{ HFD } \\
\hline & ND & - & FCC 3\% & FCC $5 \%$ & $\mathrm{SV} 10 \mathrm{mg} / \mathrm{kg}$ \\
\hline \multicolumn{6}{|l|}{ Body weight (g) } \\
\hline Baseline & $32.8 \pm 0.53$ & $31.1 \pm 0.28$ & $33.2 \pm 1.29$ & $31.7 \pm 1.68$ & $32.5 \pm 1.90$ \\
\hline Final & $44.2 \pm 3.19$ & $64.4 \pm 4.44^{\# \#}$ & $45.4 \pm 5.41^{* *}$ & $44.4 \pm 3.24^{* *}$ & $55.2 \pm 3.82^{* *}$ \\
\hline Weight gain (g) & $11.42 \pm 2.85$ & $33.23 \pm 4.05^{\# \#}$ & $12.20 \pm 4.82^{* *}$ & $12.72 \pm 2.98^{* *}$ & $22.66 \pm 2.27^{* *}$ \\
\hline \multicolumn{6}{|c|}{ Tissue weight (g/kg body weight) } \\
\hline Spleen & $3.45 \pm 0.09$ & $2.68 \pm 0.08^{\#}$ & $2.61 \pm 0.13$ & $3.04 \pm 0.09^{*}$ & $2.52 \pm 0.16$ \\
\hline Liver & $36.20 \pm 0.55$ & $34.70 \pm 3.61$ & $31.38 \pm 3.11$ & $33.30 \pm 3.88$ & $30.76 \pm 3.10$ \\
\hline Epididymal fat pad & $10.72 \pm 1.27$ & $43.02 \pm 1.34^{\# \#}$ & $37.10 \pm 1.79^{*}$ & $11.25 \pm 0.63^{* *}$ & $49.43 \pm 0.78$ \\
\hline
\end{tabular}

ND; normal chow diet, HFD; high fat diet, FCC; fermented mixture of Chaga and Cheonggukjang with L. acidophilus KCTC 3925 . SV; simvastatin (10 mg/kg body weight) treatment on HFD mice. \# and \#\# indicate $p<0.05$ and $p<0.01$ compared with the ND group, respectively; ${ }^{*}$ and ${ }^{* *}$ indicate $p<0.05$ and $p<0.01$ compared with the HFD group, respectively.

developed using enhanced horseradish peroxidase-conjugated anti-rabbit secondary antibodies. Blots were re-probed with anti$\beta$-actin antibody as a control for protein loading. Bands were visualized with the EZ-Western Lumi Pico reagent (Daeil Lab., Korea) according to the manufacturer's instructions.

\section{Statistical Analysis}

Data were presented as mean \pm standard deviation (SD). Statistical significance in the differences between control and experimental values was calculated by analysis of variance (ANOVA) using the Tukey test, and was performed using GraphPad Prism 5 (GraphPad Software, USA). Significant values are denoted by hashes and asterisks $\left({ }^{\#} p<0.05\right.$ and ${ }^{\#} p<0.01$ compared with the ND group, and ${ }^{*} p<0.05$ and ${ }^{* *} p<0.01$ compared with the HFD group).

\section{Results}

\section{Effect of FCC on Body Weight and Organ Weight in the HFD Induced-Obesity Model}

During the 12-week experiment, there was no treatmentrelated mortality or behavioral changes such as salivation, lethargy, and tremors at at any given dose. The effects of FCC on body weight and organ weights are shown in Table 2. There were no significant differences in baseline body weights among the groups. After 12 weeks, the weight gain of the HFD group was 2.9-fold higher than that of the ND group $(p<0.01)$. The mice in the FCC3, FCC5, and SV groups showed significant suppression of body weight gain compared with the mice in the HFD group.
A

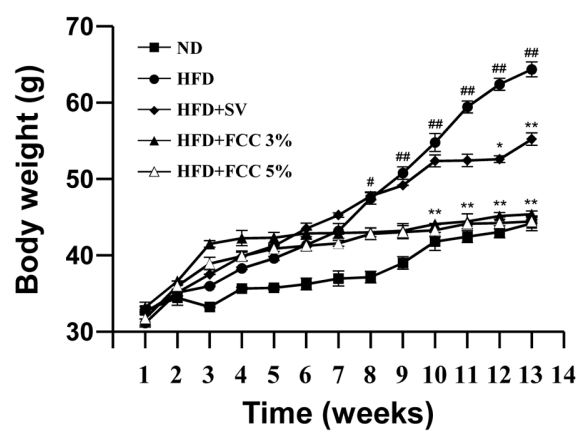

B

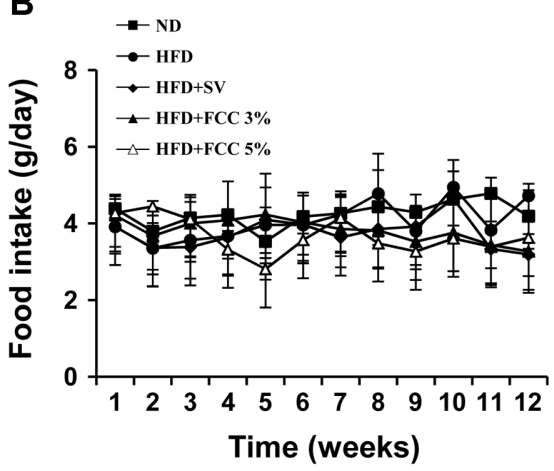

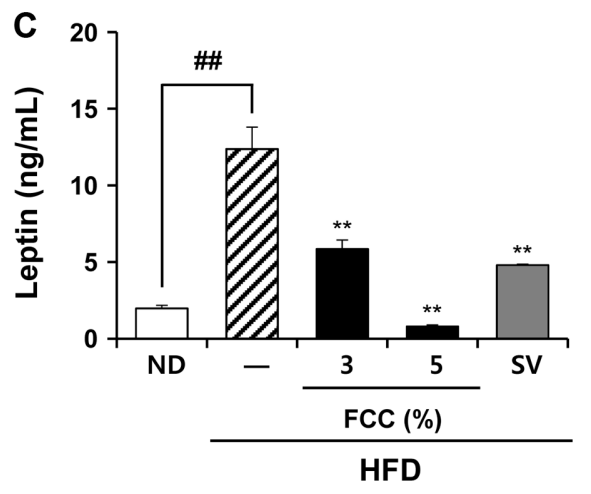

Fig. 1. Effects of FCC on body weight change, rate of food uptake and serum leptin levels.

Male ICR mice were fed ND and HFD in the presence or absence of SV, 3\% and 5\% FCC for 12 weeks ( $n=10$ per group). Changes in body weight (A) and rate of food uptake (B) were measured weekly. After 12 weeks, serum leptin levels were determined using a commercial assay kit (C). Values represent the mean $\pm \mathrm{SD} .{ }^{*} p<0.05$ and ${ }^{\# \#} p<0.01$ compared with the ND group; ${ }^{*} p<0.05$ and ${ }^{* *} p<0.01$ compared with the HFD group. ND, normal diet; HFD, high-fat diet; FCC; secondary extract of the mixture of Cheonggukjang and Chaga mushroom extract fermented by L. acidophilus KCTC 3925; SV, simvastatin (10 mg/kg body weight). 
FCC3 and FCC5 both prevented weight gain more effectively than SV treatment (Table 2 and Fig. 1A). In conjunction with the significant increase in body weight, mice in the HFD group showed a significant accumulation of epididymal fat (4-fold), but a decrease in spleen weight $(22.3 \%)$, compared with the ND group (Table 2). The liver weight of HFD mice was slightly decreased compared with that of ND mice, but was not statistically significant. SV treatment significantly suppressed the increase in body weight but did not suppress epididymal fat accumulation (Table 2). FCC3 and FCC5 intake both significantly suppressed the accumulation of epididymal fat mass.

In cases of obesity, the size of adipocytes is increased due to the accelerated storage of TG in the adipose tissue. The increase in epididymal fat weight may be associated with enlargement of fat tissue. Qualitative evaluation of the morphological changes in epididymal fat through H\&E staining revealed that HFD caused marked enlargement of fat tissue in the epididymis, but this effect was suppressed in the FCC3 and FCC5 groups (Fig. 2A). These results indicate that FCC supplementation suppresses the increase in adipocyte size, resulting in an associated reduction in adipose tissue. In accordance with the weaker suppression of epididymal fat mass by SV (Table 2), SV treatment was less effective in suppressing the enlargement of fat in the epididymal tissue than FCC supplementation (Fig. 2A).

\section{Effect of FCC on the Body Weight, Rate of Food Uptake and Serum Leptin Levels}

FCC intake significantly decreased body weight gain in HFD-fed mice (Fig. 1A). Gain or loss of body weight may be associated with the rate of food uptake. There was no significant difference in the rate of food uptake among the FCC- or SV-treated groups (Fig. 1B). This result suggests that the suppression of body weight gain caused by FCC intake is not associated with the reduction in the rate of food uptake, but with the potential anti-obesity effects of FCC. Obesity initially leads to the secretion of leptin to increase energy metabolism [7]. HFD significantly increased leptin levels, but FCC supplementation significantly reduced the increase in leptin levels in HFD mice (Fig. 1C). Because serum leptin levels were determined at the end of experiment, it is unclear whether FCC supplementation led to the suppression of blood leptin level during the experimental period. However, leptin levels are closely related to body weight and thus, decreased leptin levels indirectly reflect a reduced body weight in the FCC groups than in the HFD group.

\section{Effect of FCC on Serum Lipid Levels in HFD Mice}

Twelve-week intake of HFD resulted in significant increases in TG (Fig. 3A), T-Chol (Fig. 3B), and LDL-C (Fig. 3C) levels. SV treatment significantly suppressed the

(A) Epididymal fat pad
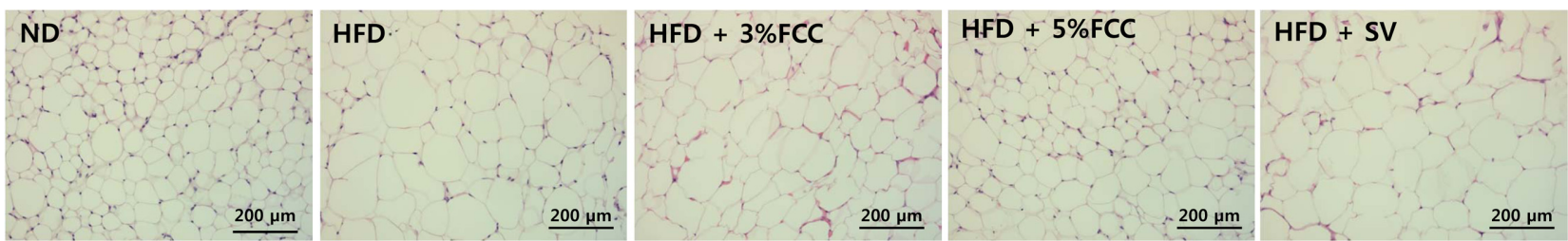

(B) Liver
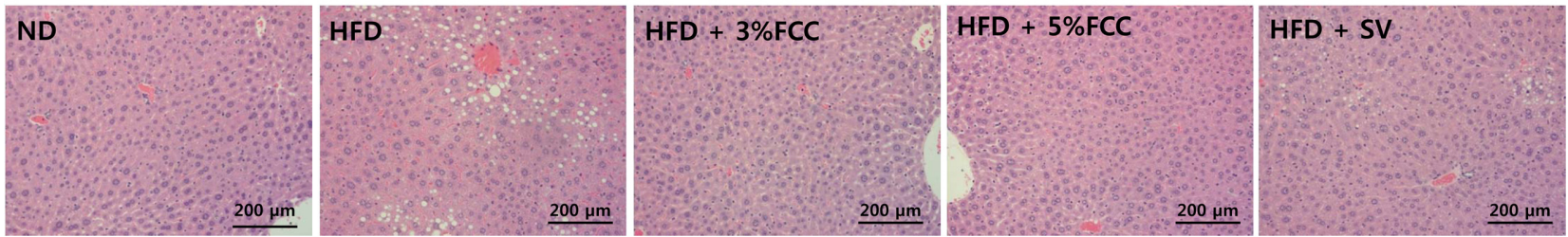

Fig. 2. Histology of epididymal adipose tissue and liver.

Representative microphotographs of mouse epididymal fat pad (A) and liver (B). Male ICR mice were fed ND and HFD in the presence or absence of SV, $3 \%$ and $5 \%$ FCC for 12 weeks ( $n=10$ per group). Fresh epididymal adipose tissue was fixed in $10 \%$ formalin, embedded in paraffin, sectioned at a thickness of $5 \mu \mathrm{m}$ and stained with hematoxylin and eosin (H\&E). All images are shown at 200× magnification. ND, normal diet; HFD, high-fat diet; FCC; secondary extract of mixture of Cheonggukjang and Chaga mushroom extract fermented by L. acidophilus KCTC 3925; $\mathrm{SV}$, simvastatin (10 $\mathrm{mg} / \mathrm{kg}$ body weight). 

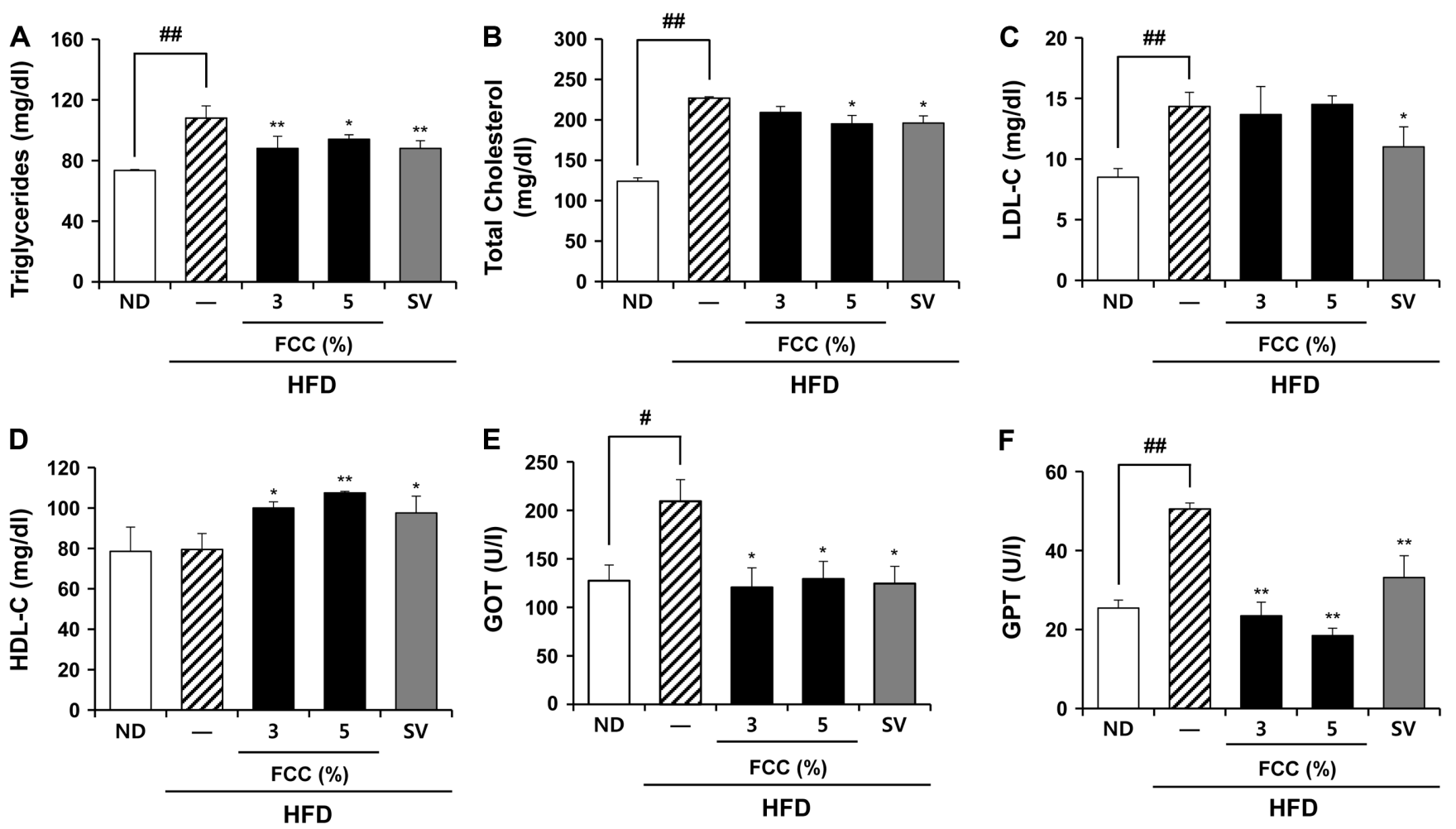

Fig. 3. Effect of FCC on the blood lipid profiles, and GOT and GPT levels.

After 12 weeks, triglyceride (A), total cholesterol (B), LDL-C (C), HDL-C (D), GOT (E), and GPT (F) levels were determined by enzymatic methods. ${ }^{\#} p<0.01$ compared with the ND group; ${ }^{*} p<0.05$ or ${ }^{* *} p<0.01$ compared with the HFD group. Values represent mean $\pm \operatorname{SD}(n=10$ per group). ND, normal diet; HFD, high-fat diet; FCC; secondary extract of the mixture of Cheonggukjang and Chaga mushroom extract fermented by L. acidophilus KCTC 3925; SV, simvastatin (10 mg/kg body weight); LDL-C, low-density lipoprotein-cholesterol; HDL-C, high-density lipoproteincholesterol; GOT, aspartate aminotransferase; GPT, alanine aminotransferase.

increase of TG, T-Chol, and LDL-C. FCC supplementation significantly reduced the serum levels of TG and T-Chol in HFD mice, but did not reduce LDL-C. In contrast to SV treatment, FCC supplementation increased the serum level of HDL-C (Fig. 3D) in HFD mice. These results suggest that intake of FCC will be beneficial in reversing the undesired changes in serum lipid profiles caused by HFD.

\section{Effect of FCC on Serum Levels of GOT and GPT in HFD- Fed Mice}

The high uptake of fat associated with obesity causes fatty liver. Thus, we evaluated the effect of FCC supplementation on fat accumulation in the liver through H\&E staining (Fig. 2B). Unlike in the ND group, the HFD group had abundant lipid droplets in the liver, which may indicate excessive accumulation of fat. Although there were no significant differences in liver weight among the HFD-fed groups (Table 2), HFD mice showed a marked increase in lipid droplets and the increased lipid accumulation in the liver was attenuated by FCC3 and FCC5 supplementation (Fig. 2B). Increased blood lipid levels caused by HFD may impair liver function through accumulation of fat in the liver. Along with accumulation of lipid droplets in the liver, the levels of GOT (Fig. 3E) and GPT (Fig. 3F) were significantly increased in HFD mice compared with those in ND mice. However, FCC treatment resulted in a significant decrease of GOT $(p<0.05)$ and GPT $(p<0.01)$ levels.

\section{Effect of FCC on Liver Histology and mRNA Expression Level of COX-2 in HFD-Fed Mice}

COX-2 is an important mediator in the process of liver inflammation. HFD mice showed a significant increase in the expression levels of COX-2 mRNA in the liver (Fig. 4A, $p<0.01$ ). FCC supplementation (FCC3 and FCC5) prevented the increase in COX-2 mRNA exhibited by HFD mice. This suppression of COX-2 upregulation seemed to be beneficial in the alleviation of hepatic inflammatory response. 

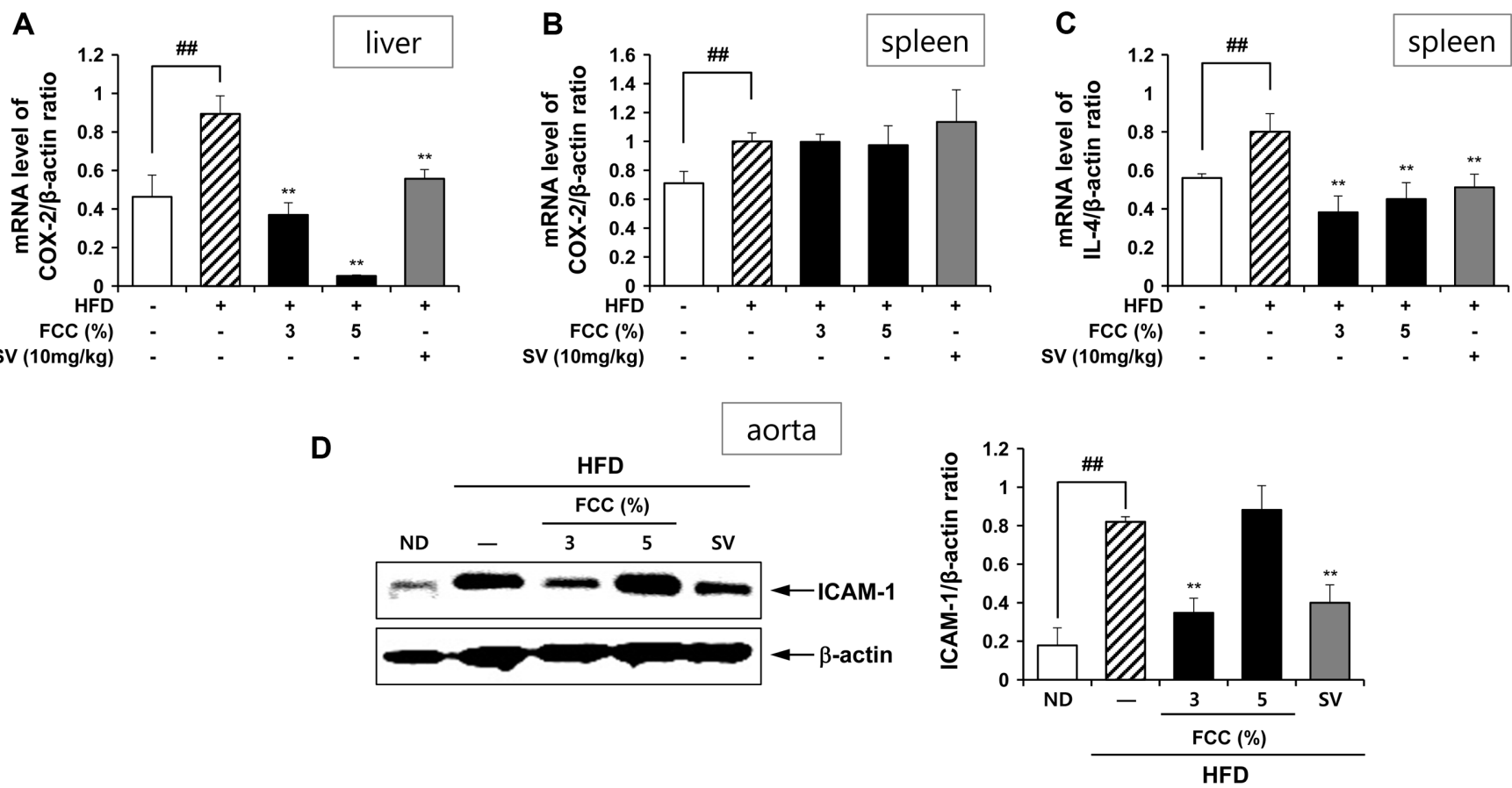

Fig. 4. Changes in COX-2 mRNA level in the liver, COX-2 and IL-4 mRNA levels in the spleen, and ICAM-1 protein level in the aorta.

After 12 weeks, the liver, spleen, and aorta tissues were removed from the mice. The mRNA expression levels of: hepatic COX-2 (A), splenic COX2 (B), and splenic IL-4 (C) were quantified by qRT-PCR. Values represent the relative ratio to $\beta$-actin as a mean \pm SD $(n=10$ per group). The protein expression levels of ICAM-1 in the aorta were quantified by immunoblotting. ${ }^{\#} p<0.01$ compared with the ND group; ${ }^{* *} p<0.01$ compared with the HFD group. ND; normal chow diet, HFD; high-fat diet, FCC; secondary extract of the mixture of Cheonggukjang and Chaga extract fermented by L. acidophilus KCTC 3925; SV, simvastatin (10 mg/kg body weight).

\section{Effect of FCC on mRNA Expression Level of COX-2 and IL-4 in the Spleen}

As shown in Table 2, HFD caused significant loss of splenic tissue weight. Thus, we investigated the effect of FCC on the mRNA expression level of COX-2 (Fig. 4B) and IL-4 (Fig. 4C), an important proinflammatory Th2-related cytokine. Similar to the expression in the liver, the mRNA expression level of COX-2 was significantly increased in the spleen of HFD mice $(p<0.01)$. The mRNA expression levels of IL-4, which plays an important role in regulating allergic responses and inflammatory effects, were also significantly increased in HFD mice (Fig. 4C, $p<0.01$ ). Although FCC or SV did not cause any changes in splenic COX-2 levels, both caused significant reductions in the mRNA expression levels of IL-4 in the spleen. These results suggest that HFD may cause excessive IL-4 production in the spleen, which can be inhibited by FCC. It was not immediately clear why spleen weight was reduced in HFD mice but excessive production of COX-2 and/or IL-4 may play a part.

\section{Effect of FCC on Protein Expression Levels of ICAM-1 in the Aorta}

ICAM-1 is an important mediator of vascular inflammation, which can be stimulated by obesity [5]. As shown in Fig. 4D, HFD mice showed a significant upregulation of ICAM-1 expression (4-fold increase) in the aorta. However, this increase of ICAM-1 was significantly suppressed by FCC3 intake. For reasons that are unclear, FCC5 did not show any reduction in the expression level of ICAM-1, and this may need further elucidation in future experiments.

\section{Discussion}

In this study, we demonstrated that FCC supplementation as a $3 \%($ FCC 3$)$ or $5 \%$ (FCC5) fraction of total diet is beneficial in the prevention of weight gain, lowering of lipid profiles, and reduction of fat accumulation in HFDmediated obesity. In addition, FCC supplementation can suppress HFD-mediated inflammatory response in the liver via suppression of COX-2 expression, in the aorta via 
suppression of ICAM-1 expression, and in the spleen via suppression of IL-4 production.

Excessive weight and obesity ultimately result from an excess of energy intake (diet) relative to energy expenditure. With an increasing prevalence of overweight or obesity across all age groups, dietary supplementation and use of herbal remedies to achieve weight loss has become a major focus for improving public health in many countries. There are several classes of anti-obesity drugs: 1) modulators of monoamine neurotransmission; 2) gastrointestinal and pancreatic peptides that regulate food intake; and 3) peripheral modulators of digestion, metabolism, and lipogenesis [7]. Owing to the multi-causal etiology and complex pathophysiology of obesity-associated complications, obesity should be treated with well-chosen drug combinations rather than with a single drug [7], and treatment with multi-target herbs will be more effective in both preventive and therapeutic approaches than with a single compound $[26,27]$. Practically, the individual intake of chaga mushrooms and cheonggukjang for the purpose of obesity control is inconvenient, because preparing optimal amounts of each and consuming them with appropriate timing is not easy on a day-to-day basis. To obtain the best synergistic effect between chaga mushroom and cheonggukjang, the two should be taken at the same time or prepared as a mixture. FCC supplementation produced anti-obesity effects and resulted in the reduction of body weight and fat mass, and in improved blood lipid profiles. Weight loss can be achieved by targeting and controlling specific molecules crucial for energy balance modulation [7]. FCC was effective in inhibiting weight gain induced by a HFD diet (Fig. 1A and Table 2). SV treatment was more effective in reducing blood lipid profiles than FCC supplementation. However, FCC intake was more effective in the suppression of weight gain and epididymal fat pad. The reduction of weight gain in HFD mice via FCC supplementation was not caused by reduced food intake as intake rate was consistent across the groups. Thus, it can be assumed that FCC intake may be exerting its effect by influencing energy metabolism and lipid deposition in the epididymal fat pad and liver tissue. HFD-mediated impairment of liver function and accumulation of fat in the liver was significantly ameliorated in the presence of FCC. Although the LDL-C lowering effect was not significant, the serum level of HDL-C was significantly augmented in HFD mice by FCC supplementation (Fig. 3D). The increase in HDL-C levels by FCC suggests it may be useful as a dietary supplement in subjects treated with TG- or LDL-C-lowering medication.

The lowering of lipid profiles is not the only primary method by which the negative effects of obesity can be alleviated. The suppression of inflammatory responses is also promising, because obesity-associated chronic inflammation can eventually cause damage to the blood vessels, liver, and spleen. COX-2 plays a role in the progression of non-alcoholic fatty liver in HFD-induced obesity but a COX-2 inhibitor (e.g. nimesulide) treatment attenuates hepatic inflammation [28]. IL-4 plays a central role in allergic inflammatory condition, and the inhibition of IL-4 through monoclonal antibodies has been clinically tested [29]. The suppressive effect of FCC on IL-4 production may be associated with the immunomodulatory action of chaga extract, because chaga extract reduced the production of IL-4 and suppressed allergic reaction in ovalbuminsensitized mice [30]. FCC supplementation decreased the mRNA expression levels of COX-2 gene in the liver and significantly suppressed the upregulated expression of IL-4 gene in the spleen (Fig. 4). This result is considered a Th2predominant effect caused by the addition of chaga mushroom extract, which means that cheonggukjang supplemented with chaga may exert enhanced anti-allergy and anti-atopy effects. FCC supplementation also attenuated the aortic expression of ICAM-1 proteins, the excessive expression of which may be a risk factor in blood vessel inflammation. Based on the findings in this study, the regular intake of FCC may be beneficial in controlling highfat diet-mediated transition of obesity. However, the underlying mechanism of FCC on the suppression of tissue inflammatory markers is currently unclear.

Cheonggukjang is considered an excellent source of proteins, lipids, amino acids, vitamins, and minerals, and is commonly used for health benefits $[11,13,31]$. The antiobesity effects of cheonggukjang intake is mediated through its interference with the adipogenesis process in the liver and adipose tissues by down-regulating the expression of primary adipogenic transcription factors [32]. Using health-promoting bacteria, there have been numerous efforts to develop functional foods with enhanced characteristics such as taste, bioavailability, and healthpromoting potential, compared with the classic forms [33]. It is difficult to compare our current study directly with previous publications on the anti-obesity effects of cheonggukjang, chaga extract and chaga-cheonggukjang due to the differences in diet formulation, animal species, and treatment duration [4,34-38]. The enhanced functional benefits of cheonggukjang may be associated with the presence of isoflavones [39], which are actively involved in mitigating metabolic diseases, and their bioavailability can be increased with the aid of fermentation [40, 41]. 
Isoflavone aglycone-rich diet can reduce liver and serum total cholesterol levels, and liver triglyceride levels in cholesterol-fed rats [42]. Our previous study demonstrated that FCC possesses $28 \%$ more isoflavone aglycones than unfermented chaga-cheonggukjang [24]. In addition, chaga extract contains water-soluble melanin complex, which plays a role in improving insulin sensitivity, and reducing adiposity in HFD mice [43]. It is also effective in the improvement of fat metabolism through the regulation of adiponectin levels [44]. Considering the health-promoting potential of isoflavone aglycones [39], it can be assumed that FCC would be superior to general cheonggukjang in controlling obesity, because FCC may possess combinatorial effects from both cheonggukjang and chaga extracts which may regulate either the same or different targets in the various pathways associated with obesity [4, 34-38] and inflammation [12, 18, 45-47].

Collectively, FCC supplementation attenuated obese conditions caused by high-fat diet and suppressed inflammatory responses in the liver and spleen. The underlying molecular mechanisms involved in the anti-obesity effect of FCC and the combinatorial effects of cheonggukjang and chaga as related to the levels of isoflavone and aglycone should be investigated in further studies.

\section{Acknowledgments}

This study was supported by the Business for Cooperative R\&D between Industry, Academy, and Research Institute funded by the Korea Small and Medium Business Administration in 2016 (Grant No.C0442776).

\section{Conflict of Interest}

The authors have no financial conflicts of interest to declare.

\section{References}

1. Hotamisligil GS, Shargill NS, Spiegelman BM. 1993. Adipose expression of tumor necrosis factor-alpha: direct role in obesity-linked insulin resistance. Science 259: 87-91.

2. Hotamisligil GS. 2006. Inflammation and metabolic disorders. Nature 444: 860-867.

3. Duan Y, Zeng L, Zheng C, Song B, Li F, Kong X, et al. 2018. Inflammatory links between high fat diets and diseases. Front. Immunol. 9: 2649.

4. Ganesan K, Xu B. 2018. Anti-obesity effects of medicinal and edible mushrooms. Molecules 23: 2880.

5. Koh KK, Han SH, Quon MJ. 2005. Inflammatory markers and the metabolic syndrome: insights from therapeutic interventions. J. Am. Coll. Cardiol. 46: 1978-1985.
6. Mitchell NS, Catenacci VA, Wyatt HR, Hill JO. 2011. Obesity: overview of an epidemic. Psychiatr. Clin. North. Am. 34: 717-732.

7. Konstantinos L, Karavis M, Mastorakos G, Valsamakis G. 2000. New Molecular Targets for the Pharmacotherapy of Obesity, pp. 24-25. In Feingold KR, Anawalt B, Boyce A, Chrousos G, Dungan K, Grossman A, Hershman JM, Kaltsas G, Koch C, Kopp P, Korbonits M, McLachlan R, Morley JE, New M, Perreault L, Purnell J, Rebar R, Singer F, Trence DL, Vinik A, Wilson DP (eds.), Endotext, Ed. MDText.com, Inc., South Dartmouth (MA).

8. Karr S. 2017. Epidemiology and management of hyperlipidemia. Am. J. Manag. Care. 23: S139-s148.

9. Coward L, Barnes NC, Setchell KDR, Barnes S. 1993. Genistein, daidzein, and their beta-glycoside conjugates: antitumor isoflavones in soybean foods from American and Asian diets. J. Agric. Food Chem. 41: 1961-1967.

10. Ramdath DD, Padhi EM, Sarfaraz S, Renwick S, Duncan AM. 2017. Beyond the cholesterol-lowering effect of soy protein: a review of the effects of dietary soy and its constituents on risk factors for cardiovascular disease. Nutrients 9: 324.

11. Mun EG, Kim B, Lee EY, Lee HJ, Kim Young, Park Y, et al. 2018. Research trend in traditional fermented foods focused on health functional evaluation. J. Korean Soc. Food Sci. Nutr. 47: 373-386.

12. Cho CW, Han CJ, Rhee YK, Lee YC, Shin KS, Shin JS, et al. 2015. Cheonggukjang polysaccharides enhance immune activities and prevent cyclophosphamide-induced immunosuppression. Int. J. Biol. Macromol. 72: 519-525.

13. Yang HJ, Park S, Pak V, Chung KR, Kwon DY. 2011. Fermented soybean products and their bioactive compounds, pp. 21-58. In El-Shemy H (eds.), Soybean and Health, Ed. InTech, Shanghai

14. Shin SK, Kwon JH, Jeong YJ, Jeon SM, Choi JY, Choi MS. 2011. Supplementation of cheonggukjang and red ginseng cheonggukjang can improve plasma lipid profile and fasting blood glucose concentration in subjects with impaired fasting glucose. J. Med. Food. 14: 108-113.

15. Bae MJ, Shin HS, See HJ, Chai OH, Shon DH. 2014. Cheonggukjang ethanol extracts inhibit a murine allergic asthma via suppression of mast cell-dependent anaphylactic reactions. J. Med. Food. 17: 142-149.

16. Lee JH, Paek SH, Shin HW, Lee SY, Moon BS, Park JE, et al. 2017. Effect of fermented soybean products intake on the overall immune safety and function in mice. J. Vet. Sci. 18: 25-32.

17. Glamočlija J, Ćirić A, Nikolić M, Fernandes Â, Barros L, Calhelha RC, et al. 2015. Chemical characterization and biological activity of Chaga (Inonotus obliquus), a medicinal "mushroom". J. Ethnopharmacol. 162: 323-332.

18. Mishra SK, Kang JH, Kim DK, Oh SH, Kim MK. 2012. Orally administered aqueous extract of Inonotus obliquus ameliorates acute inflammation in dextran sulfate sodium (DSS)-induced colitis in mice. J. Ethnopharmacol. 143: 524-532.

19. Shin YK, Son HU, Kim JM, Heo JC, Lee SH, Kim JG. 2015. Cinnamomum cassia bark produced by solid-state fermentation 
with Phellinus baumii has the potential to alleviate atopic dermatitis-related symptoms. Int. J. Mol. Mede. 35: 187-194.

20. Kim OK, Chang JY, Nam DE, Park YK, Jun W, Lee J. 2015. Effect of Canavalia gladiata extract fermented with Aspergillus oryzae on the development of atopic dermatitis in NC/Nga mice. Int. Arch. Allergy Immunol. 168: 79-89.

21. Rodríguez H, Curiel JA, Landete JM, de las Rivas B, López de Felipe F, Gómez-Cordovés C, et al. 2009. Food phenolics and lactic acid bacteria. Int. J. Food Microbiol. 132: 79-90.

22. Kim JS, Lee JH, Surh J, Kang SA, Jang KH. 2016. Aglycone Isoflavones and exopolysaccharides produced by Lactobacillus acidophilus in fermented soybean paste. Prev. Nutr. Food Sci. 21: 117-123.

23. Kim JY, Nolte LA, Hansen PA, Han DH, Ferguson K, Thompson PA, et al. 2000. High-fat diet-induced muscle insulin resistance: relationship to visceral fat mass. Am. J. Physiol. Regul. Integr. Comp. Physiol. 279: R2057-2065.

24. Na HG, Jang EH, Nam DH, Kim MA, Kim MJ, Sohn EH, et al. 2018. Physicochemical properties and isoflavone content of Chaga Cheonggukjang with Lactobacillus acidophilus KCTC 3925 starter. Korean J. Food Preserv. 25: 221-228.

25. Livak KJ, Schmittgen TD. 2001. Analysis of relative gene expression data using real-time quantitative PCR and the 2(Delta Delta C(T)) method. Methods 25: 402-408.

26. Wagner H. 2011. Synergy research: approaching a new generation of phytopharmaceuticals. Fitoterapia 82: 34-37.

27. Yang Y, Zhang Z, Li S, Ye X, Li X, He K. 2014. Synergy effects of herb extracts: pharmacokinetics and pharmacodynamic basis. Fitoterapia. 92: 133-147.

28. Tsujimoto S, Kishina M, Koda M, Yamamoto Y, Tanaka K, Harada Y, et al. 2016. Nimesulide, a cyclooxygenase-2 selective inhibitor, suppresses obesity-related non-alcoholic fatty liver disease and hepatic insulin resistance through the regulation of peroxisome proliferator-activated receptor gamma. Int. J. Mol. Med. 38: 721-728.

29. Catley MC, Coote J, Bari M, Tomlinson KL. 2011. Monoclonal antibodies for the treatment of asthma. Pharmacol. Ther. 132: 333-351.

30. Ko SK, Jin M, Pyo MY. 2011. Inonotus obliquus extracts suppress antigen-specific IgE production through the modulation of Th1/Th2 cytokines in ovalbumin-sensitized mice. J. Ethnopharmacol. 137: 1077-1082.

31. Jeong PH, Shin DH, Kim YS. 2008. Effects of germination and osmopriming treatment on enhancement of isoflavone contents in various soybean cultivars and cheonggukjang (fermented unsalted soybean paste). J. Food Sci. 73: H187-194.

32. Kim J. 2017. Anti-obesity and anti-inflammation effects of Cheonggukjang in C57Bl/6 mice with high fat diet induced obesity. J. Life Sci. 27: 1357-1368.

33. Douillard FP, de Vos WM. 2019. Biotechnology of healthpromoting bacteria. Biotechnol. Adv. 19: 30044-30048.

34. Soh JR, Shin DH, Kwon DY, Cha YS. 2008. Effect of Cheonggukjang supplementation upon hepatic acyl-CoA synthase, carnitine palmitoyltransferase I, acyl-CoA oxidase and uncoupling protein 2 mRNA levels in C57BL/6J mice fed with high fat diet. Genes Nutr. 2: 365-369.

35. Kim J, Choi JN, Choi JH, Cha YS, Muthaiya MJ, Lee CH. 2013. Effect of fermented soybean product (Cheonggukjang) intake on metabolic parameters in mice fed a high-fat diet. Mol. Nutr. Food Res. 57: 1886-1891.

36. Choi JH, Pichiah PB, Kim MJ, Cha YS. 2016. Cheonggukjang, a soybean paste fermented with B. licheniformis-67 prevents weight gain and improves glycemic control in high fat diet induced obese mice. J. Clin. Biochem. Nutr. 59: 31-38.

37. Kim MS, Kim B, Park H, Ji Y, Holzapfel W, Kim DY, et al. 2018. Long-term fermented soybean paste improves metabolic parameters associated with non-alcoholic fatty liver disease and insulin resistance in high-fat diet-induced obese mice. Biochem. Biophys. Res. Commun. 495: 1744-1751.

38. Joo JI, Kim DH, Yun JW. 2010. Extract of Chaga mushroom (Inonotus obliquus) stimulates 3T3-L1 adipocyte differentiation. Phytother. Res. 24: 1592-1599.

39. Zaheer K, Humayoun Akhtar M. 2017. An updated review of dietary isoflavones: Nutrition, processing, bioavailability and impacts on human health. Crit. Rev. Food Sci. Nutr. 57: 1280-1293.

40. Lee DH, Kim MJ, Ahn J, Lee SH, Lee H, Kim JH, et al. 2017. Nutrikinetics of isoflavone metabolites after fermented soybean product (Cheonggukjang) ingestion in ovariectomized mice. Mol. Nutr. Food Res. 61: 1700322.

41. Taku K, Umegaki K, Sato Y, Taki Y, Endoh K, Watanabe S. 2007. Soy isoflavones lower serum total and LDL cholesterol in humans: a meta-analysis of 11 randomized controlled trials. Am. J. Clin. Nutr. 85: 1148-1156.

42. Kawakami Y, Tsurugasaki W, Nakamura S, Osada K. 2005. Comparison of regulative functions between dietary soy isoflavones aglycone and glucoside on lipid metabolism in rats fed cholesterol. J. Nutr. Biochem. 16: 205-212.

43. Lee JH, Hyun CK. 2014. Insulin-sensitizing and beneficial lipid-metabolic effects of the water-soluble melanin complex extracted from Inonotus obliquus. Phytother. Res. 28: 1320-1328.

44. Kim B, Kim MS, Hyun CK. 2016. Suppression of adiposity and improvement of fat metabolism in high-fat diet-induced obese mice treated with an Inonotus obliquus extract. Kor. J. Pharmacogn. 47: 172-178.

45. Choi J, Kwon SH, Park KY, Yu BP, Kim ND, Jung JH. Chung HY. 2011. The anti-inflammatory action of fermented soybean products in kidney of high-fat-fed rats. J. Med. Food. 14: 232-239.

46. Kim YR. 2005. Immunomodulatory activity of the water extract from medicinal mushroom Inonotus obliquus. Mycobiology 33: 158-162.

47. Nguyet TMN, Lomunova M, Le BV, Lee JS, Park SK, Kang JS, et al. 2018. The mast cell stabilizing activity of Chaga mushroom critical for its therapeutic effect on food allergy is derived from inotodiol. Int. Immunopharmacol. 54: 286-295. 